\title{
Development of a System for Detecting Abnormal Vibration during High-Speed Winding
}

\author{
HASEGAWA Katsumi, TABEI Tadashi, ${ }^{* *}$ HIKITA Shinichi, ${ }^{* *}$ SUZUKI Tamotsu* \\ and HORIKA WA Takeshi \\ "Toray Engineering Co. Ltd., Sonoyama, Otsu 525-0842, Japan, $\quad$ "Toray Co. Ltd., Nihonbashi, Tyuo-ku, Tokyo \\ 103-0022, Japan $\quad{ }^{* * *}$ RYUKOKU University, Seta, Otsu 520-2141, Japan
}

Based on the Textile Machinery Society of Japan, Vol.53, No.4,T93-T106(2000)

\begin{abstract}
The purpose of this research is to propose a new system, for detecting abnormal vibration during high-speed winding. The system gives a warning when the abnormality vibration of a winder has reached a certain level, or stops only the winder with the problem before the winding yarn package is broken.

The research analyzes the data obtained from the operating winder, and compares with data acquired from he overhauled winders that were re-assembled and re-adjusted. The results of the analysis have validated the effectiveness and practicability of the system that systematically detects the level of abnormality in the winders to prevent a costly accident. And it is possible to adopt the Preventive Maintenance of winders by the Condition Based Maintenance System based on this system.
\end{abstract}

Key Words: Preventive maintenance of winders; Detection of abnormal vibration of winder; Breakage of winding yarn package

\section{Introduction}

The winding speed of the POY/DTY machines, which started to be generally used in the latter half of 1970 s, ranged from 3,000 to 3,500 meters per minute. In the high oriented yarn process using one of the latest processing methods, the speed has increased up to 6,000 meters per minute. It would be highly possible in the near future that the processing speed will become higher.

In such an ultra-high speed processing of yarn, much higher reliability shall be required for the winders and related equipment. Furthermore, since the winders shall be provided with a device that allows bobbins to be easily set up or taken off from them, a variety of problems have come to be noticed in order to provide high-speed stability and strict maintenance.

A yarn-winding machine has a variable rotation number; from beginning of winding to end, the number of rotation gradually decreases but the load of quantity of yarn to be wound increases steadily. The vibration value during the yarn winding process is, therefore, not constant. Furthermore, when the spindle of the fully-wound package stops, a big vibration will occur because today's winders equipped with an automatic yarn change device are operated above their sympathetic vibration value range ${ }^{1)}$.

From these reasons, conventional vibration monitoring systems for such rotary machinery with a small load variation and almost constant number of rotation cannot beapplied to filament yarn winders. Therefore, we have expected more reliable new monitoring systems that can judge the causes that input vibration value becomes large: for example, substantially deteriorated winders and operational conditions.

\section{Construction of Winder's Vibration Monitoring System}

\subsection{Vibration of Filament Yarn Winders}

A vibration value of a winder in the filament yarn winding process may change depending on a variety of conditions involved, and it shall never be constant. It may be affected, for example, by the following conditions.

1) The primary cause of changing vibration values in the filament yarn winding process is a ribbon winding ${ }^{2)}$. Normally a filament yarn is properly distributed and wound on the surface of a package. Whenever the ribbon-winding pattern appears, a filament yarn is piled up on the same thread guide several times. The filament yarn is, therefore, wound in a thick and solid state like a cord, generating irregularities on intersections of the patterns, thus causing vibration. The number of ribbon windings that appears during the filament yarn winding process is theoretically quite large.

2) Then, the vibration value also increases when the number of spindle rotation gradually decreases and comes to sympathize with the frequency at the resonance point of a large number of component parts such as roller bail, a traversing device, frames, covers, and members of an auto doffing device consisting of a winder.

3) In addition, in the case of a filament yarn winder

\footnotetext{
* Corresponding author E-mail:ccs65070@syd.odn.ne.jp
} 
equipped with an automatic doffing device, the number of spindle rotations will traverse the sympathetic frequencies of both the supporting frame of a spindle shaft and a spindle shaft when a fully wound package stops. In such occasions, the winder also vibrates significantly.

Figure 1 shows an example of the vibration values caused by these factors during the filament yarn winding process. A filament yarn was wound at the speed of 3,265 meters per minute with a six-degree helix angle. The axis of abscissa shows the number of spindle rotations, and the axis of ordinate shows a vibration rate. It may be considered that a large vibration near $2,700 \mathrm{rpm}$ seen near the end of winding and a slightly larger vibration near $5,400 \mathrm{rpm}$ are due to a ribbon winding. It is considered that another large vibration near $6,000 \mathrm{rpm}$ is caused by a different factor.

As mentioned above, since winders in the filament yarn winding process include a variety of vibration factors, their vibration values fluctuate in various ways.

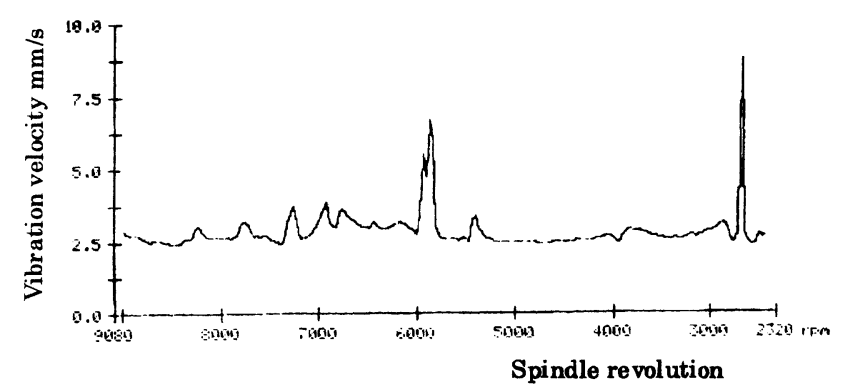

Fig.1 Vibration amplitude of a winder during winding

\subsection{Vibration Information to be Taken}

As mentioned above, a vibration value of rotary machinery and equipment represents their own condition. When its condition is good enough, and there is no problem in the machinery, the vibration value is kept small. However, as the condition deteriorates, their vibration value will gradually increase. The filamentit yañ winding machine is fundamentally the same as other rotary machinery. However, in the case of the filament yarn winder, since some specific problems are entangled, it is necessary to remove such factors and to take out only such vibration information that is essentially caused by the sequential change. Then, we have studied the vibration information to be taken into the system.

When we try to engage a filament yarn onto a bobbin, especially when a roller bail that helps flatten a yarn layer makes direct contact with the bobbin and rotates together, both the noise and vibration are quite large. When the layer has reached thickness of several millimeters when both the noise and vibration come to be significantly still. At this moment, in the winder equipped with an auto doffing device, rotation of a spindle on the fully wound package side has already stopped. Therefore, when no ribbon winding phenomenon appears in several minutes after the filament yarn was engaged on the bobbin, there is less external noise, and the vibration condition well represents the characteristics of a spindle itself. As mentioned above, although a number of variable factors depending on the bobbin's chucked state are involved, this moment may be considered to be optimal in terms of repeatability of vibration conditions and reliability of vibration values.

Concerning variable factors by the chuck condition of a bobbin, since it is impossible to remove them altogether, a certain variable range should be accepted. However, the variation is in a certain state, even though there exists a minute variable trend, it may be buried by the variable range. So, it is impossible to grasp such a trend. For this problem, we have considered that when we examine a changing trend of the average value for a certain period, even if there is a variable factor in each cycle, we will be able to remove it and grasp a minute variable trend.

\subsection{Monitoring Specifications of the System}

We have studied abnormal conditions for the filament yarn winders that can be monitored by the system. First of all, there is a long-term machinery deterioration that has been generating in the winders over a long period of time. Above all, the following phenomena are typical.

1) Should the holding function of a bobbin chuck deteriorate, the eccentric quantity of a bobbin may increase, thus the imbalance quantity of the spindle and vibration as well increasing.

2) A bearing that supports a high-speed rotation of a spindle shaft, particularly, its housing with which the bearing's outer race is engaged may wear and tear. In the spindle shaft, since its inner race rotates, and the engagement between the shaft and the bearing is made solid, abrasion of the part hardly occurs. However, since the engagement with the housing is rather loose, a phenomenon that the outer race may move in the opposite direction to the rotating direction of the spindle shaft can be quite often observed. Hence, the housing is likely to wear. Should any abrasion occur, it may cause the spindle to run out, thus increasing the vibration. We may clearly determine whether or not the vibration was caused by these reasons by anaiyzing the vibration wave forms using a FFT. If the vibration was caused by one of these reasons, we may easily confirm that the vibration of frequency that follows the spindle rotation is generating, because a maximum vibration value may appear at the position of the rotation frequency.

3) Then, there is another problem that the bearing is flawed. Should the defect grow bigger, it may lead to a serious accident because the bearing will seize up during rotating. The flaw of bearing may affect the vibration wave forms. The vibration frequency generated by the flaw can be calculated using the following formula ${ }^{3)}$.

Should the outer race flaw,

$$
Z f_{c}=Z \times \frac{1}{2} f_{0}\left(1-\frac{d}{D} \cos \alpha\right)
$$

Should the inner race flaw,

$$
Z f_{i}=Z \times \frac{1}{2} f_{0}\left(1+\frac{d}{D} \cos \alpha\right)
$$

Should the rolling element flaw, 


$$
Z f_{b}=f_{0} \times \frac{D}{d}\left[1-\left(\frac{d}{D}\right)^{2} \cos ^{2} \alpha\right]
$$

Here, $\mathrm{Z}$ is the number of balls, $f_{0}$ is the rotation frequency of spindle shaft, $D$ is diameter of the pitch circle of the anti-friction bearing, $d$ is diameter of the rolling element of the anti-friction bearing, and $\alpha$ is contact angle.

If we will try to analyze whether or not there is actually any flaw in the bearing from the vibration information, we may use the following method.

Should the flaw of the bearing make contact with or hit the rolling element, an impact force will be generated. The force then causes the outer or inner race of the anti-friction bearing to generate a shock vibration at the frequency of its characteristic vibration. Therefore, in order to select a wave form due to the flaw of any bearing, a band-pass filter shall be used. Since the above-mentioned shock vibration is cyclically generated every time when the rolling element passes through the flaw on the bearing, the wave form through the band-pass filter shall be provided with an envelop processing, then it will be transformed into a cyclical wave form every time when the element passes through the flaw. Then, both a peak to peak value $A$ after such processing (High peak: Should the value be big enough, the flaw exists in the rolling element) and a value $B$ of the one-cycle wave form area (Should the value be big enough, the flaw is a total abrasion flaw) shall be calculated. If the wave form after the envelop processing should be provided with the FFT analyzing, an outstanding wave form may be observed at the position of the frequency corresponding to the above-mentioned calculated values in the case that there exists a flaw on the bearing.

Among the possible events, there is an unexpected package collapse during the filament yarn winding process. Although it rarely happens, once it should happen, it would lead to a serious accident. This is a kind of phenomenon that occurs when a plurality of multi-filament yarns are wound simultaneously; one of the filaments flows into the adjacent filament yarn, mixed with it, and then is wound continuously. A yarn package consisting of a small number of filaments is likely to be wound softer. On the contrary, a package consisting of a large number of filaments is wound harder. Should the softness exceed such a limit that can keep the package intact, the package would be suddenly broken by a great centrifugal force. The force may cause the spindle to stop rapidly, leading to an accident where the spindle is broken.

The phenomenon has been said to occur abruptly. When it is, however, observed from the vibration point of view, a gradual increase in the vibration values can be seen in the initial deformation stage of a package. Therefore, it may be possible for us to prevent such accidents by catching and pre-processing the phenomenon before it occurs.

These kinds of phenomena may become the major items that can be grasped by the winder's vibration monitoring system, although there may be some difference between long-term factors and short-term ones.

\subsection{Demonstration of the System}

\section{1) Construction of the System}

The schematic of the system shall be as follows. Individual winders shall be equipped with the vibration pick-up sensors that can detect the vibration level. A local station (L/S) that controls about thirty units of winders shall take the vibration information from these pick-up sensors. The station shall then process the taken wave forms into a predetermined shape, and compare them with the monitored values that were determined by an abnormal vibration monitoring comparator. Should there be no problem, both the processed values and wave forms shall be sent to the center station where such information is internally managed

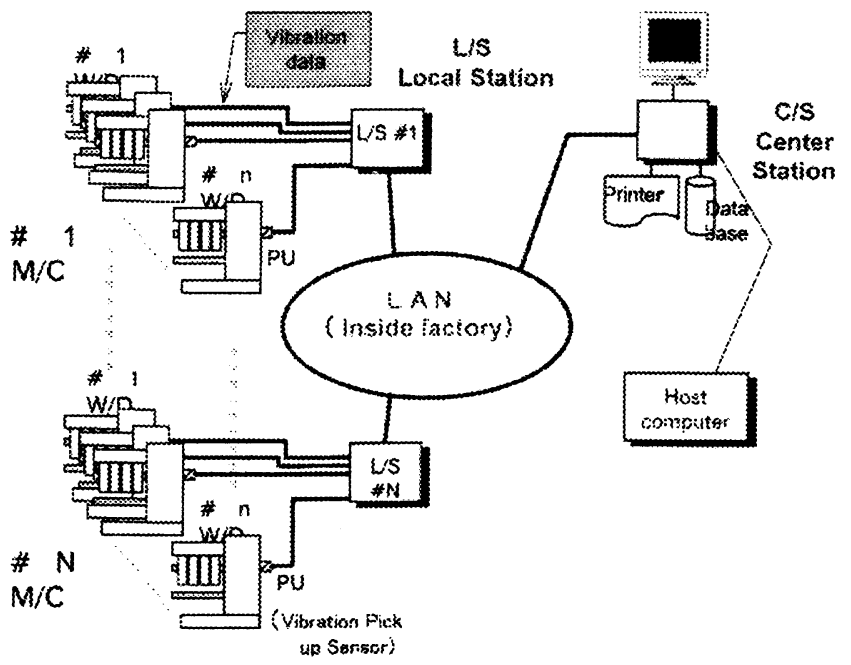

Fig.2 Schematic of system architecture

by way of LAN, and stored into the database. Figure 2 shows a schematic of the high-speed winder's abnormal vibration monitoring system. The winder groups with winders \#1 to \#n as a winder group shall be \#1 M/C to \#N $\mathrm{M} / \mathrm{C}$, and individually different types of filament yarn to be produced shall be allocated to each of such groups. Each winder shall be equipped with an accelerated vibration pick-up sensor P. For a position to which the sensor shall be set, since a winder equipped with an auto-doffing device has such a structure that two revolving drums with two spindles rotate every time when a package is doffed, it is impossible to provide the spindle with the vibration pick-up sensor directly. Then, we made tests to decide which position was optimal to pick up the vibration of the spindle, and have decided that the sensor shall be located on the frame beside the revolving drum (Fig. 3). In order to detect a package

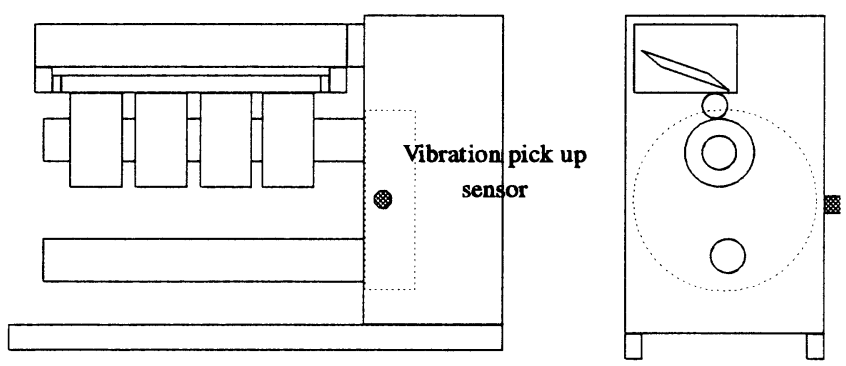

Fig.3 Position of the vibration pick-up sensor 
collapse in the winding process well before it actually occurred, it is necessary to monitor the vibration condition of each winder in a short time. We have allocated thirty-two units of winders (This number will not be necessarily fixed) to a local station. Individual winders shall be connected to one of the local stations, L/S \#1 to L/S \#N.

Meanwhile, when a package doffing signal shall be transmitted from the host computer that controls individual winders' winding time, to both the winder control panel board and center station, it will be conveyed to a local station via the center station. Then the local station shall take in the vibration information of the winders at a given time after the doffing. Such information shall be converted into a content that will be mentioned later, and compared with a predetermined abnormal vibration monitoring alarm value. Then whenever necessary, appropriate measures shall be taken. After that, such information shall be sent to the center station (C/S) that internally controls information via the LAN in the plant, and then stored into the database.

Should there be any abnormal vibration, it shall be transmitted to the center station, and alarm signals shall be transmitted therefrom. Whenever necessary, operators can access the vibration information stored in the data-base, check the vibration trend of each winder or bearing, and then grasp the real situations of the winders where the alarm signals were transmitted.

\section{2) Information Processing at the Local Station}

Figure 4 shows how the data are processed at each local station. Five minutes after the local station receives the package doffing information from the center station, the local station shall connect Switch (1) shown in the figure to the applicable winder. At that time Switch (2) has been connected to the circuits of Channel 1 and 2 .

The obtained vibration information shall be divided into Channel 1 and Channel 2, and at the former the received vibration acceleration information shall be converted into vibration velocity information. Then peak to peak value $C$ shall be calculated. The value $C$ shall be compared with a predetermined abnormal vibration monitoring value $\mathrm{C}_{0}$. If formula $\mathrm{C}_{0}>C$ should be formed, both the values $C$ and the wave form converted into a digital value shall be sent to the center station by way of the LAN, and then they shall be stored into the database. Should formula $\mathrm{C}_{0} \quad C$ be formed, the fact that abnormal events have occurred shall be sent to the center station, together with the transmission of the data.

In Channel 2, the received vibration acceleration information shall be, first of all, processed by the band-pass filter. The winders use two kinds of bearings $\# 6009$ and \#6010. Since the frequency of vibration wave form transmitted by these bearings shall be intermediate values between $10 \mathrm{kHz}$ and $20 \mathrm{kHz}, 10 \mathrm{kHz}$ to $20 \mathrm{KHz}$ band-pass filters shall be used. The wave forms through such band-pass filters shall be given an envelope process. Both the peaks to peak value $A$ (High peak) and the wave form area value $B$ (High average) for one cycle period shall be calculated from the resultant wave form. After that, these values $A$ and $B$ shall be compared with the predetermined abnormal vibration monitoring alarm values $\mathrm{A}_{0}$ and $\mathrm{B}_{0}$. If $A \leq \mathrm{A}_{0}$ or $B<\mathrm{B}_{0}$, values $A, B$ and the envelop processed wave form shall be transmitted to the center station by way of the LAN, and stored into the data base. If $A \quad \mathrm{~A}_{0}$ or $B \quad \mathrm{~B}_{0}$, the information that abnormal events have occurred shall be conveyed to the center station together with the data.

Since a periodical vibration monitoring shall be made once when a package is doffed, the work for it may be finished in a short time. The local station can, therefore, allocate the remaining time for checking thirty-two units of winders in turn every one second. At this time, Switch (1) shall change one by one every one second and Switch (2) shall be connected to Channel 3 .

The vibration information taken into Channel 3 every thirty-two seconds shall be converted into the peak to peak value $C$, and compared with abnormal vibration monitoring set value $\mathrm{D}_{0}$ by the abnormal vibration monitoring comparator. If $C<\mathrm{D}_{0}$, these values shall be stored into the data-base of the applicable winder. Therefore, the vibration information obtained every thirty-two seconds shall be stored in each local station. In the meantime, if $C \quad \mathrm{C}_{0}$, the information that abnormal events have occurred shall be given to the center station, and value $C$ shall be stored into the data-base. The value $C$ stored every thirty-two seconds shall be stored for five hours, and when a new datum is stored into the data-base, the old value $C$ shall be erased.

In the case that abnormal data continue twice, we have provided the particular winder with a function to automatically stop the rotation of the spindle. The measure will help prevent such an accident that a package will collapse, leading the spindles to stop suddenly.

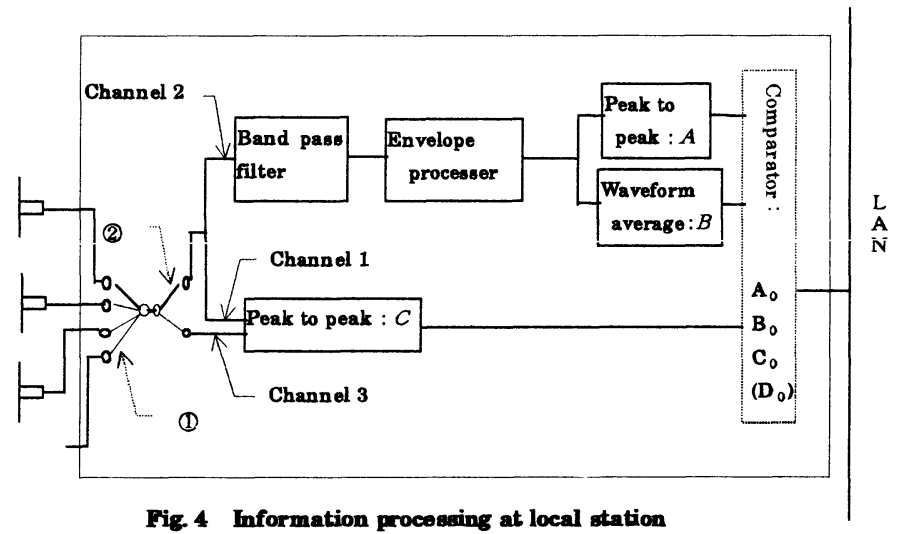

\section{3) Measurement of Winders' Vibration}

In order to confirm the practical serviceability of the developed system, we have tentatively applied the system to the sixteen units of winders equipped with an auto doffing device that have been operated for the past ten odd years. At the same time, we have also gathered a variety of vibration data to analyze the current conditions of such winders.

Among the winders, we have selected two characteristic winders; One of which is \#I winder. Although it is small in terms of spindle's rotation with vibration, it has a possibility that there may be a flaw of its bearing. Another is \# II winder which is largest in terms of spindle's rotation with vibration. 
The vibration charts were sampled from these winders.

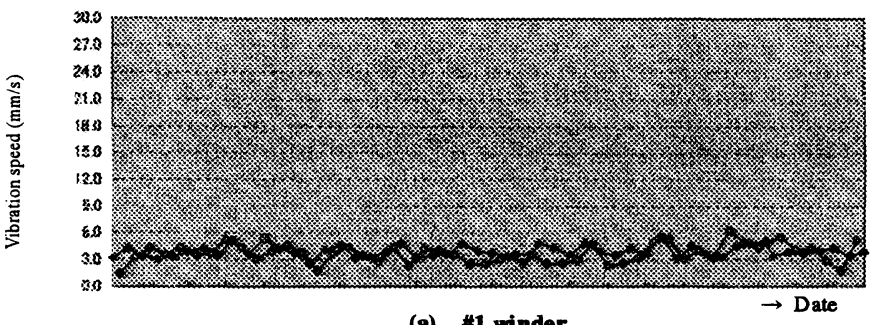

(a) \#1 winder

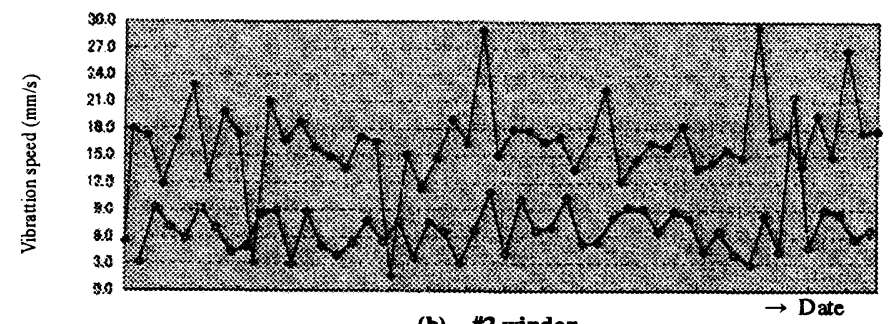

(b) $\$ 2$ winder

Fig.5 Vibration amplitude of the winder at 5 minutes after yarn change

\section{(1) Imbalance and Rotation with Vibration}

Figure 5 (\#I, \#II) shows a data chart that the System has so far collected and recorded. The figure consists of an axis of abscissa as a time axis, and an axis of ordinate that shows a vibration speed in order of package doffing, representing a transition of values for about a month period. $\diamond(\mathrm{spA})$ and $\checkmark$ (spB) marks show two spindles' vibration values. Since package doffing shall be made between two spindles $\diamond$ (spA) and $\diamond(\mathrm{spB})$ interchangeably, $\diamond$ and $\diamond$ marks shall be shown one by one sequentially. Although the vibration values always show going up and down at the beginning of yarn winding, the variation may be regarded due to the change of eccentricity amount. Both spA and spB of \#I show a stable transition of vibration values. Contrarily, in the spB of \#II a certain abnormality can be seen in terms of variation of vibration values and their pattern. Although operation hours for \#I and \#II have been the same, it has now become clear that there is a big difference between them in vibration conditions.

Figure 6 shows a trend of vibration amplitude for \#I winder, where a mean value of the machine's vibration for a month is represented by one point, and the values for six months are plotted. Although the spindle has shown a good condition for six months, the speed of vibration increases monotonously only for

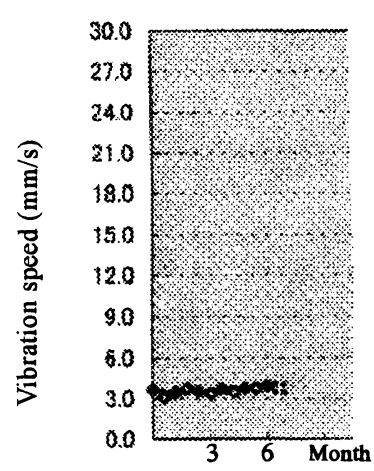

Fig.6 Trend of vibration amplitude plotted by the mean value for a month such a short period.

\section{2) Bearings' Flawing}

Let us explain the collected data on the bearings' flawing.

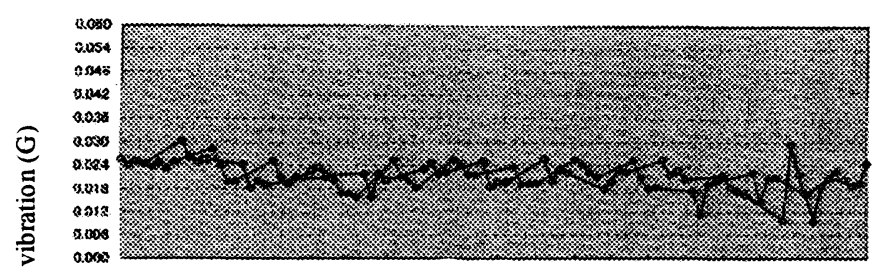

(a) High average in \# I winder

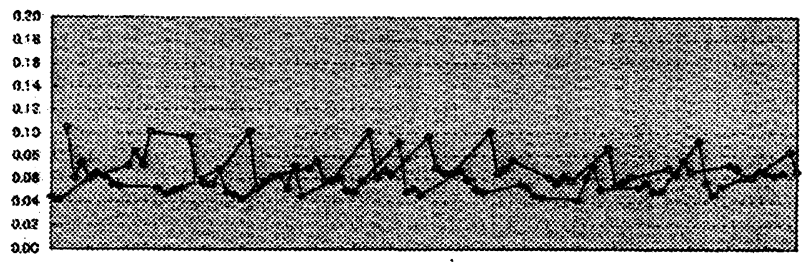

(b) High peak in \# I winder

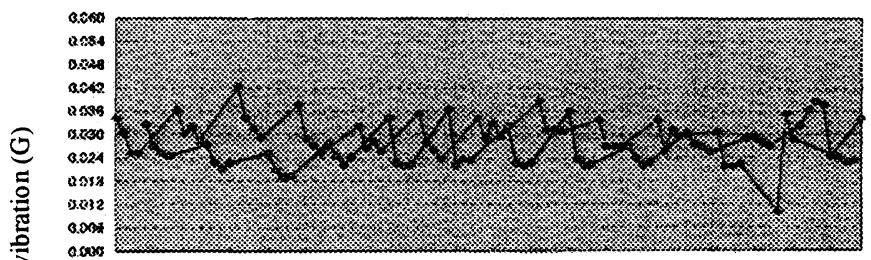

(c) High average in \# II winder

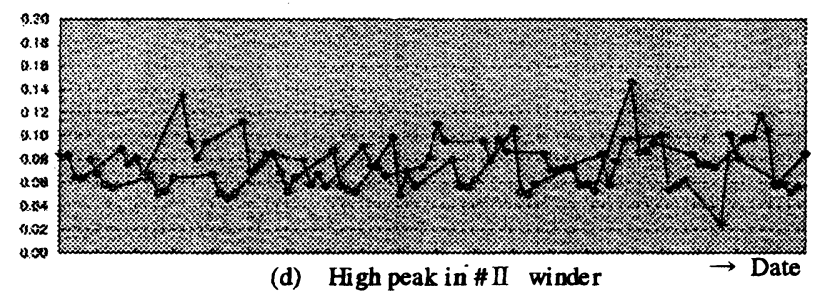

Fig.7 Charts of High average and High peak on the bearing

Figure 7 shows four different charts ((a) High average in \# I winder, (b) High peak in \# I winder, (c) High average in \# II winder and (d) High peak in \# II winder) of the High average and High peak for \#I winder and \#II winders, respectively. The axis of abscissas shows a time and axis of abscissa shows vibration acceleration. The values sampled every 5 minutes after the yarn change shall be plotted in the charts. The reason why four continuous data are plotted in the charts is that the vibration data were sampled five minutes after yarn change, and thereafter, the data were collected four times in every six minute cycle. This is because we have repeated tests on an assumption that depending on the timing of sampling, the vibration information due to the bearings' flawing may change.

Figure 8 shows a test result of the FFT processed High

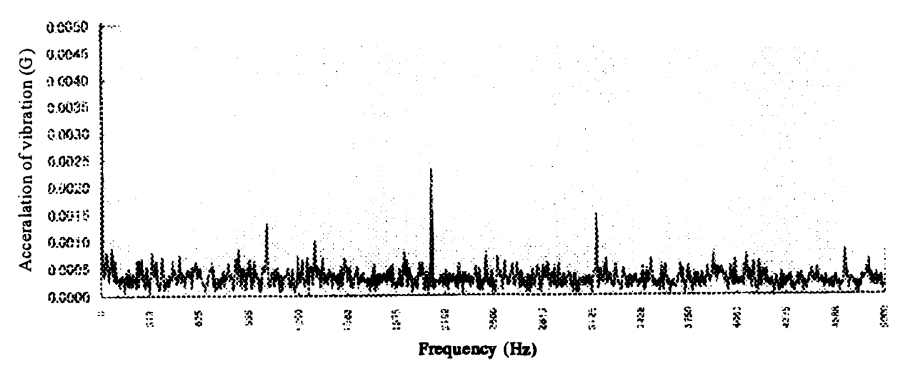

Fig.8 Frequency analyzed by the FFT on the flawed bearing 
peak value $A$ sampled five minutes after data of $\mathrm{spB}$ of \# I winder had been sampled. The axis of abscissa shows frequency, ranging 0 to $5 \mathrm{kHz}$, and the axis of ordinate shows vibration acceleration (G). The frequency showing an outstanding output corresponds to the calculated results of the bearings' flawed outer races (Not only a peak frequency of approximately $1 \mathrm{kHz}$ but also twice or even three times higher peak frequencies are shown here). Since the FFT analyzed result of the data sampled at other points are different in conditions under which the information was taken in. Hence the frequency does not show the completely same pattern.

Figure 9 is the FFT processed frequency chart of the data taken in five minutes after the data of spB in \#II winder had been taken. Since the outstanding peak frequency shown here is quite different from the frequency released from the flawed bearing, it is necessary to investigate the cause in the future.

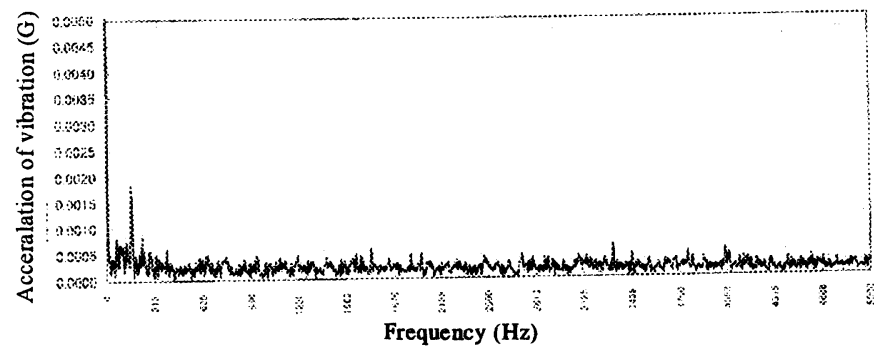

Fig.9 FFT of frequency different from hurt bearing frequency

\section{(3) Abnormal Vibration during Yarn Winding}

Vibration values are sampled every thirty-two seconds for each winding machine. The vibration data for five-hour operation have been always stored at the local station. Figure 10 shows an example. This chart shows a certain winder's sequential vibration values sampled every thirty two seconds during operation from the beginning time to the final time. The axis of abscissa shows a time and the axis of ordinate shows a vibration acceleration.

An effective use of this function will allow us to make the following program for the winders: In the early stage of winding operation in which a package collapse occur, its vibration value begins to rise, and when the value exceeds a monitoring alarm value, that point will be caught by the program. If continuous two value exceeds, it would stop the spindle. The chart also reveals that a change in the package diameter, or the change in the number of spindle rotations will change the vibration value of the winder in many ways.

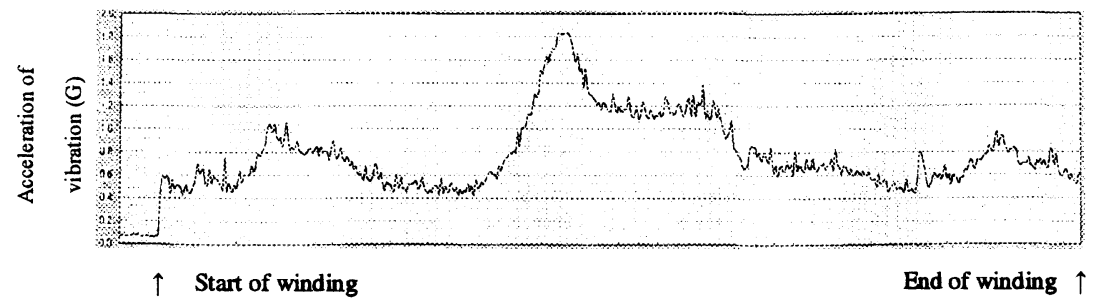

Fig.10 Vibration amplitude picked up by the system
Several charts in Figures $5 \sim 10$, concerning various vibration amplitudes, have been sampled from the used winders. It is quite interesting to know how these data reflect the current conditions of the operating winders, and what sort of behaviors will be seen for the winders that have started operation. Since these problems also are important, we have disassembled winders for detail investigation. The following is a result.

\section{Investigated Results of Disassembled Winders}

Before we start investigation of disassembled winders, we have studied what do these vibration charts mean. We can suppose from spA for \#II winder in Figure 5, that a deteriorated chuck mechanism has weakened its chuck force, causing a bobbin to be eccentrically set when it is set on the spindle, thus increasing its imbalance amount. Otherwise, the members around the bearing have abraded, causing the spindle to rotate with vibration, resulting in the increased imbalance. Next, from Figures 7 and 8, it is supposed that corresponding to the simulated result when the bearings have flawed, the outer race of the $\# 6010$ bearings has flawed in four bearings used for the \#I winder's spB. Furthermore, from Figure 9, we have supposed that the \#II spB has abnormal factors other than bearings; for example, there is some clearance around the bearing, and then, there is such a possibility that vibrations may occur at a peculiar frequency.

Table 1 Inepection results on the bearing

\begin{tabular}{|c|c|c|c|c|c|}
\hline \multirow{2}{*}{\multicolumn{2}{|c|}{$\begin{array}{l}\text { W/D No. } \\
\text { Spindle }\end{array}$}} & \multicolumn{2}{|c|}{ \# I } & \multicolumn{2}{|c|}{ \# II } \\
\hline & & sp A & sp B & sp A & sp B \\
\hline \multirow{4}{*}{$\begin{array}{c}\text { Brg } \\
\text { position } \\
\text { (from top) }\end{array}$} & $\# 1$ & 0 & 0 & 0 & $\Delta$ \\
\hline & $\# 2$ & 0 & $\star$ & $\Delta$ & $\star$ \\
\hline & $\# 3$ & $\Delta$ & 0 & $\Delta$ & $\Delta \sim \star \star$ \\
\hline & $\# 4$ & $\star$ & $\Delta$ & 0 & 0 \\
\hline
\end{tabular}

\subsection{Bearings}

When we disassembled the bearings, we discovered a viscous gelled substance containing brown-black oil on the bearings and their peripherals, which can also be seen on the disassembled general machinery and equipment. It was, therefore, expected that the bearing sections abraded. We removed bearings out of the spindle using a jig without damaging the spindle, and we rotated it supporting the bearings by hands after we washed them with oil. Then we inspected the vibration condition. Table 1 shows the results.

On the table, two bearings \#1 and \#2, are located in the tip section built into the cylindrical section of the spindle, and \#3 is a bearing supporting the rear part of the spindle. These bearings are of \#6010 type. \#4 is a bearing of \#6009 type located in the rear section of the driving motor. We had a roughly shocked feeling when we rotated the bearings marked $\star$ by hands.

We are now under way to request the 
bearing maker to make a detail investigation.

\subsection{Abrasion of the Shaft and the Housing at the} Bearing Section

Then, we measured the abrasion level of the spindle shaft and the housing. Table 2 shows the result. Since we have passed members within the drawing allowance in the manufacturing stage without mentioning individual dimensions, detail dimensions of each member manufactured remain unknown. However, since we always aim at the median of the allowance in manufacturing, we have enclosed values that were out of the drawing allowance.

In the case of the inner race rotation, since the spindle shaft and the bearing are solidly engaged together, no abrasion is seen in the shaft section. However, a slight abrasion could be seen in one out of four shafts. However, in the bearing's housing section, since its engagement allowance is loose, abrasion is easy to occur; abrasion of three spindles of four have progressed out of the manufacturing allowance. At the bearing positions of \#4 winder, there can be seen a lot of abrasion phenomena in a large number of spindle shafts. At the position where there exists a serious abrasion, even a tip of finger was able to feel roughness. It is supposed that generation of this sort of gap has resulted in the abnormal output that does not correspond with the calculated values shown in Figure 9.

From these analytical results, it has become possible to understand the vibration information data more widely.

Table 2 Abrasion at the shaft and the housing around bearings

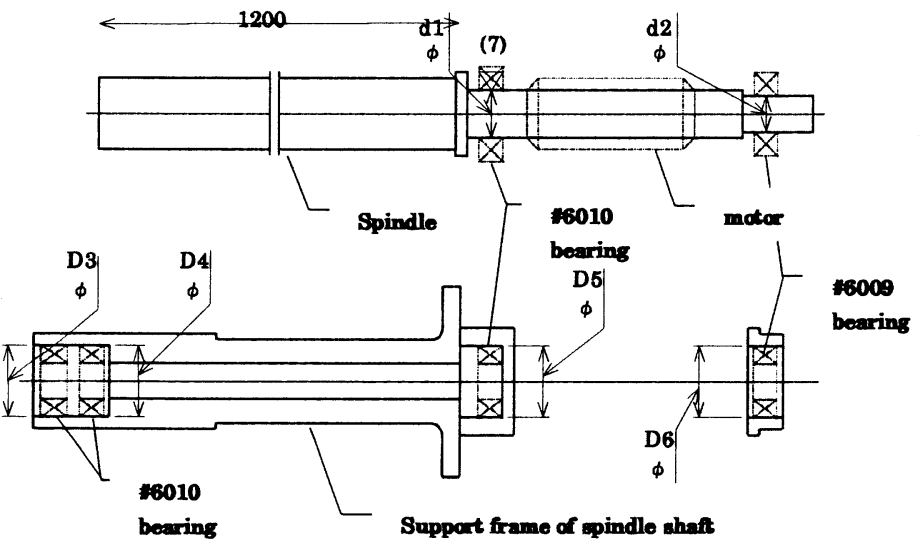

\begin{tabular}{|c|c|c|c|c|c|c|}
\hline & & \multirow{2}{*}{$\begin{array}{c}\text { Drawing } \\
\text { dimension }\end{array}$} & \multicolumn{2}{|c|}{ 亘I } & \multicolumn{2}{|c|}{ \#II } \\
\hline & & & SpA & Sp B & $\mathrm{SpA}$ & $\mathrm{Sp} B$ \\
\hline \multirow[t]{2}{*}{$\phi \mathrm{d} 1$} & $x-x$ & \multirow{2}{*}{$\phi 50 \begin{array}{l}+0.013 \\
+0.002 \\
\end{array}$} & 050.004 & -50.002 & 1.499998 & $\$ 50.004$ \\
\hline & $Y-Y$ & & ${ }_{6} 50.003$ & $\phi 50.002$ & 649999 & $\phi 50.005$ \\
\hline \multirow[t]{2}{*}{$\phi d 2$} & $x-x$ & \multirow{2}{*}{$\begin{array}{c}\phi 45 \\
+0.013 \\
+0\end{array}$} & ه45.002 & $\$ 45.003$ & $\$ 45.004$ & $\$ 45.005$ \\
\hline & $Y-Y$ & & \$45.002 & C.45.002 & 645.003 & ه4.004 \\
\hline \multirow[t]{2}{*}{${ }_{\phi}$ D3 } & $x-x$ & \multirow{2}{*}{$680^{+0}$} & $\$ 80.009$ & . 800014 & 680.006 & 80.006 \\
\hline & $Y-Y$ & & $\$ 80.009$ & 6800170 & $\$ 80.006$ & $\phi 80.010$ \\
\hline \multirow[t]{2}{*}{${ }_{\phi} \mathrm{D4}$} & $x-x$ & \multirow{2}{*}{$\phi 80^{+0}$} & 680.007 & $\$ 80.012$ & $\$ 80.007$ & $\$ 80.010$ \\
\hline & $Y-Y$ & & 600.007 & 80.012 & 80.007 & 680.010 \\
\hline \multirow[t]{2}{*}{${ }_{\phi}$ D5 } & $X-X$ & \multirow{2}{*}{$680^{+0}$} & [.79.997 & 80.003 & 80.005 & 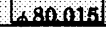 \\
\hline & $\mathbf{Y}-\mathbf{Y}$ & & 680.005 & D.79.995 & 680.000 & 800.003 \\
\hline \multirow[t]{2}{*}{${ }_{\phi} \mathrm{D} 6$} & $x-x$ & \multirow{2}{*}{${ }_{675}+0}$. & 675.000 & 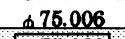 & 675.016 & 425.15 \\
\hline & $\mathbf{Y}-\mathbf{Y}$ & & ه75.011 & 676.028 & Cosona & ه75.002 \\
\hline
\end{tabular}

\section{Vibration Results after Re-assembly}

We replaced defective component parts with new parts, reassembled the spindle shafts and then readjusted them.
That is, since a very strong interest has been held in the vibration behavior of the winder of which performance is almost the same as that of the machine when operation is started, we installed it at the same place under the same conditions before it had been disassembled, and then, sampled the data.

Figure 11 shows comparative characteristics of a variety of vibrations before and after the maintenance of the winder. The left side of the figure shows various vibration characteristics of the machine before it was disassembled, and the right side shows the sampled values after it was disassembled. The positions of the winder and spindles are the same before and after the disassembly.

a) Figure 11(a) shows a transition of the periodically sampled vibration values five minutes after yarn change at the winder \#II, in which the vibration values have clearly decreased to less than one-fourth of the original ones. And the data to be compared with it before disassembly was shown in Fig. 5.

b) Figure 11(b) concerns the flawed bearings on the applicable winder spindle. In the chart, \#I winder's High average value is shown. Since all damaged bearings have been replaced when the winder was disassembled, the High average values expressing flaws of the bearings have decreased to less than one-fourth of those before it was

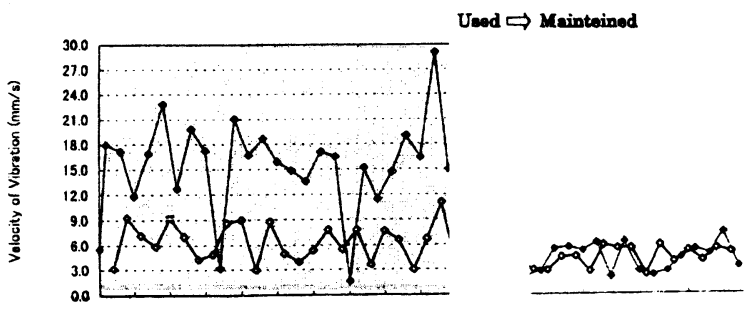

(a) Vibration amplitude of the winder every atter yarn change (* II)

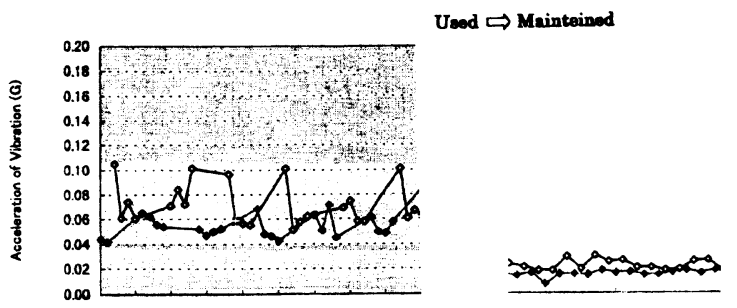

(b) Chart of High ponk value on tho boaring (* I)

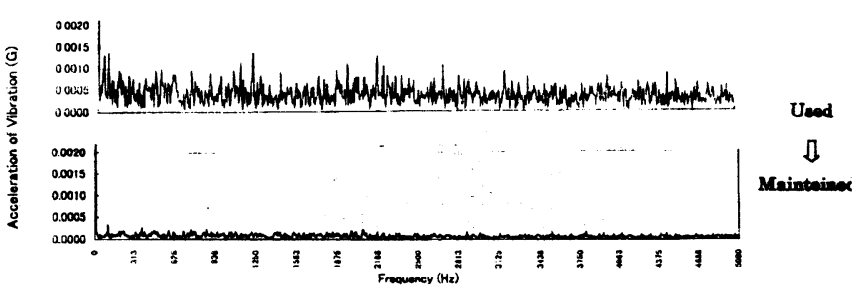

(c) Frequency analysed by the FFT an tho boaring (" I)

Fig.11 Comparative characteristics of vibration before and after the maintenance 
disassembled. The chart to be compared before disassembly is Fig. 7 for \#I winder.

c) Figure 11(c) shows the FFT results of High average values sampled in Fig. 11(b). If the chart is compared with that before disassembly, not only the vibration values have seriously decreased in the total frequency band, but also there is no specific peak wave form recognized in the chart because of the flawed bearings before the winder was disassembled. The chart to be compared shall be Fig. 8 . These chart and figure clearly show that there is a big difference between the winders before and after it was disassembled.

From the results of Fig. 11, we have confirmed that the winder, of which used bearings are replaced and spindles' imbalance is well adjusted, is small in terms of various vibration characteristics, and that the system could clearly grasp the winder's condition after it has been used for a certain period through the process that it has been worn and torn.

\section{Discussion}

\section{1) Decision of Vibration Monitored Alarm Values}

One of the vital issues for the system shall be how to set the vibration monitored alarm value. For this point, there is a literature $^{4)}$. According to it, almost a three-time larger vibration amplitude value than one for normal operation is recommended. The system adopted the same value consciously in the early stage. However, from the results such as Fig.s 5, 7 and 11, in the case of the filament yarn winder, we decided almost four to five time larger vibration monitoring alarm values than one at the time when the winder was newly manufactured because, there is still some allowance by the time when the bearing will be completely broken even after an abnormal alarm is released.

It is very important to study how the winder will be broken for how long time after its vibration amplitude exceeded the alarm value, or what sort of effect the package will undergo. However, since the study of these problems will require a lot of time and work, it will be necessary to summarize them from a large number of results, and foresee the future.

\section{2) Relations between Abnormal Imbalance Indexes and}

\section{Abnormal Bearing Indexes}

When we planned to build the system, we expected that the deteriorated bearings would be expressed by the imbalance indexes as a result. However, when we actually sampled a variety of data; and for example, when we compare Fig. 5 (\#I) with (\#II), both of which show the spindle's imbalance amount, Fig. 5 (\#II) is much larger in terms of the vibration amplitude. On the contrary, in the data of value $A$ (High peak) and $B$ (High average) in Fig. 7 that show the characteristic of the bearings, \#I is larger than \#II. This shows the fact that if a bearing should deteriorate, values $A$ (High peak) and $B$ (High average) would become large. This result will be a piece of effective information to judge future conditions of the machinery and equipment using these charts.

\section{3) Frequency Vibration Caused by Reasons Other Than Bearing Flaws}

It has been proved by a chart obtained from the maintained and re-assembled winder that the big vibration values with quite different frequency from that of the calculated values of the flawed bearing (Fig.9) have relationships with the abrasion gap in the bearing housing section.

\section{Conclusion}

A Preventive-Safety-Measurement System ${ }^{\text {s }}$ for winder spindle and maintenance system based on the winder vibration has succeeded in developing an abnormal vibration monitoring system that can take their vibration information.

A vibration value of a winder during the filament yarn winding process may change depending on the variety of conditions involved, and it shall never be stable. Therefore, the timing to take in the vibration of winder is important.

1. This monitoring system is possible for us to prevent these accidents from occurring by catching and preprocessing the phenomenon before it occurs,

1) An imbalance spindle and its rotation with vibration may further increase the vibration.

2) A flawed spindle bearing can be detected by the vibration wave form.

3) Increased vibrations that occur just before a collapsing of package would lead to a serious collapsing of the package during the winding process.

2. We have also confirmed that by setting the abnormality monitoring alarm value to four to five times larger as an appropriate value than that when the normal operation is started, within a short time after the vibration amplitude was judged abnormal, not only the winder would not reach the stop condition, but also a winder with seriously progressed abnormality can be detected.

3. Since the adoption of the system makes it possible for us to grasp the rotation conditions of a high-speed spindle, a new possibility has been generated that we can transfer the maintenance system for the winders from the conventional Time-based maintenance system to a more efficient Condition-based maintenance system. Now it is expected that the transfer will contribute to improvement of the reliability of high-speed spindles.

\section{References}

[1] K. Hasegawa, J. Text. Mach. Soc. Japan, 52, T19 (1999)

[2] K. Hasegawa, J. Text. Mach. Soc. Japan, 50, P27 (1997)

[3] Japan Plant Maintenance Association, "Equipment Diagnosis Technology", p.64, Japan Plant Maintenance Association (1990)

[4] N. Inoue; Plant Engineering, 29, No.3, P36 (1997)

[5] K. Hasegawa, "YARN-WINDING METHOD", USP $5,900,553,(1999)$ 